\title{
Lettera ai Soci
}

\section{Letter to members}

\author{
Carmine Chieffo, Carlo Vigorito, Carmine Riccio
}

Monaldi Arch Chest Dis 2008; 70: 169.

\section{Caro Socio}

In questi ultimi anni il Monaldi Archives for Chest Disease (Cardiac Series), organo ufficiale della nostra Associazione, con la sua periodicità trimestrale, ha consolidato il livello di assoluto valore che la Rivista già in precedenza aveva raggiunto nella considerazione della comunità cardiologica, nazionale. Il Monaldi è stato infatti un valido strumento per la diffusione della cultura della Cardiologia Riabilitativa attraverso i contributi scientifici originali, provenienti non solo dalle nostre strutture ma anche da ricercatori non appartenenti al GICR-IACPR, di estrema rilevanza sul piano scientifico e sempre più attinenti alle tematiche della prevenzione delle malattie cardiovascolari e della riabilitazione del cardiopatico.

La Rivista ha pubblicato risultati di linee guida, raccomandazioni e statement di estremo interesse per chi si occupa di Cardiologia Riabilitativa e Prevenzione CV secondaria, elaborati dal GICR-IACPR autonomamente $\mathrm{o}$ in collaborazione con altre società scientifiche italiane. Il Giornale ha dato inoltre evidenza anche alle survey più recenti sulla Cardiologia Riabilitativa Italiana (ISYDE e ICAROS).

Il Monaldi ti è sempre pervenuto in forma cartacea, lasciandoti nel contempo la possibilità di accesso alla edizione on-line, presente sul sito del GICR-IACPR, e di scaricare i vari contributi in forma completa. Riteniamo tuttavia che questa versione debba affiancare, ma non sostituire, l'invio del Giornale nella sua forma convenzionale cartacea: entrambi costituiscono validi mezzi di diffusione con utilizzatori e filosofia di fruizione di tipo differente.

Nel preparare l'ultimo numero del Monaldi, gli Editors ed il CD del GICR-IACPR hanno avvertito la necessità di verificare la fruibilità delle informazioni e l'efficacia della comunicazione. Abbiamo deciso pertanto di chiedere direttamente il tuo parere, pregandoti di rispondere alle domande di un questionario, inerenti non solo agli aspetti grafici e contenutistici, alle aspettative e alle abitudini dei Soci, ma soprattutto alla modalità di ricezione della Rivista: in forma cartacea o solo tramite accesso al sito.

Il questionario viene riportato nella pagina seguente ed è anche scaricabile dal sito: www.gicr.it .

Ti ricordiamo che in mancanza di una risposta specifica la rivista non ti perverrà più in forma cartacea a casa, ma la potrai consultare soltanto nel sito.

Per rivedere inoltre le liste di distribuzione della Rivista ti chiediamo di precisare con esattezza l'indirizzo di posta ordinaria (casa e/o struttura di lavoro) e le relative e-mail. Anche se tale compito prenderà qualche minuto del tuo tempo, apprezzeremo molto la tua partecipazione, che ci consentirà di programmare il numero di copie da stampare e di ottimizzarne i costi.

Avendo infine il Consiglio Direttivo stabilito, nella sua ultima riunione, di limitare l'invio della Rivista soltanto ai Soci in regola con il pagamento delle quote sociali, per evitare di essere escluso dalla lista di distribuzione del Monaldi, ti invitiamo a verificare la tua posizione associativa. A tale proposito abbiamo predisposto la modulistica, relativa all'iscrizione ed alla regolarizzazione della quota sociale, che potrai consultare nelle pagine finali di questo numero del Monaldi o scaricare dal nostro sito.

\section{Carmine Chieffo - Carlo Vigorito Editors del Monaldi}

Carmine Riccio Presidente del GICR-IACPR 


\section{Questionario per i Soci}

Cognome Nome

Via $\mathbf{N}$ CAP Città Prov

Tel. Fax E-mail

Struttura

Medico $\square$ Psicologo

Desideri ricevere il Monaldi:

In forma cartacea

Solo con accesso al sito in mancanza di una specifica risposta il Monaldi non ti sarà più inviato in forma cartacea

1) Ricevi tutti i numeri del Monaldi: 口 si a no

2) Se no, specifica quanti nell'ultimo anno:

3) Leggi il Monaldi?

- Si, tutte le sezioni

- Si, ma solo alcuni articoli

口 Raramente

4) Leggi il Monaldi perché:

$\square$ Ė utile in ambito professionale

Per conoscere le attività che si svolgono in ambito associativo

Altro, specificare

5) Quali sezioni trovi più interessanti?
$\square$ editoriali
$\square$ reviews
$\square$ articoli originali
case-report
$\square$ protocolli di studio
$\square$ notizie dalle Regioni e/o dalle Aree

6) Quali argomenti vorresti fossero approfonditi $o$ aggiunti?
7) Se abitualmente non leggi il Monaldi qual è la motivazione principale?

Non trovo le tematiche interessanti

Non è utile nella pratica lavorativa

Non ho tempo

8) Come valuti la veste editoriale e la grafica?

a Accattivante

- Piacevole

口 Non adatta

9) Consulti il sito del GICR-IACPR $w w w . g i c r . i t ?$
$\square$ Spesso
Q Qualche volta
$\checkmark$ Raramente
口 Mai

10) Leggi il Monaldi on line?
$\square$ Spesso
Qualche volta
$\square$ Raramente
प Mai

11) Hai delle considerazioni di ordine generale da esprimere?

Da inviare alla Segreteria GICR-IACPR presso:

ARISTEA International S.r.l. (all'attenzione di Carla Chiavetta)

Salita di Santa Caterina, 4 - 16123-GENOVA

Tel. 010.583224 - n. fax: 0105531544 - e-mail: segreteria@gicr.it

Il Questionario è scaricabile anche dal Sito del GICR: www.gicr.it 


\title{
Dal GICR al GICR-IACPR: nel solco della continuità e dell'innovazione
}

\section{From GICR to IACPR-GICR: continuity and innovation}

\author{
Raffaele Griffo
}

Monaldi Arch Chest Dis 2008; 70: 171-172.

U.O. Cardiologia Riabilitativa, ASL 3 "Genovese”, Ospedale La Colletta, Arenzano.

E-mail address: pastpresidente@gicr.it

Nell'ottobre del 2006, insieme agli amici del Consiglio Direttivo, tracciammo il programma del biennio 2006-2008 della nostra Società Scientifica. È stata un'operazione utile rileggerlo ora al termine del mandato per verificare quanto siano stati traguardati gli obbiettivi a suo tempo indicati o, quanto meno, ne sia stata intrapresa la strada per raggiungerli.

Cercherò quindi di fare un breve bilancio e proporvi le mie considerazioni su questi due anni di lavoro seguendo la falsariga di quel documento, sottolineando quelle iniziative che a mio parere sono state le più rilevanti. Per una dettagliata "storia" del biennio, rinvio il lettore alle periodiche newsletter pubblicate semestralmente sul sito.

\section{La politica interna}

Uno degli obbiettivi era ampliare la platea dei soci e "professionalizzare" la struttura organizzativa e scientifica del GICR. Pre-requisito essenziale a questi scopi era dotarci di una vera e propria segreteria organizzativa che tenesse le fila di una Società scientifica al passo con i tempi:l'Aristea International si è rivelata una scelta credo azzeccata, dimostrandosi un partner affidabile e motivato che ci ha assicurato non solo un supporto segretariale ed una sede prestigiosa, ma anche un adeguato advertising, supportato come al solito dall'impegno personale di tutti i componenti del Direttivo, verso l'industria per il finanziamento delle nostre iniziative di formazione ed aggiornamento, del $9^{\circ}$ Congresso Nazionale, del nostro giornale e del sito.

Successivamente a questo passaggio indispensabile, molta della nostra attenzione si è dedicata al rinnovamento e al potenziamento del sito, che grazie allo straordinario impegno di Roberto Tramarin e della sua squadra (Marco Ambrosetti, Stefania de Feo, Paola Vaghi e Segno \& Forma), è diventato non solo il portale del GICR, alimentato con grande continuità da articoli, commenti e dai resoconti delle attività del consiglio direttivo, ma anche uno strumento formidabile per la ricerca e per l'aggiornamento.
Senza di esso non sarebbero stati mai possibili ISYDE 2008, né, con altrettanta efficacia, la survey ICAROS. Il sito è diventato un vero e proprio fiore all'occhiello della nostra società, dandole un'immagine di grande vivacità, adeguata ad una società "giovane", dinamica e in forte espansione come la nostra. Credo che le sue funzionalità, insieme alle tante iniziative scientifiche e formative svolte nel biennio, siano state il vero "turbo" nel motore del GICR, a cui si devono non solo la crescita esponenziale degli iscritti (ad oggi oltre 600), ma anche una rinnovata grande intensità propositiva e culturale dei soci e una maggiore visibilità nel contesto scientifico nazionale ed internazionale.

Un altro significativo obbiettivo è stato dare una maggiore attenzione verso le aree non mediche: il Congresso Nazionale di Ostuni che ha proposto molti momenti di integrazione tra tutte le figure professionali del team riabilitativo, con il suo grande successo di attiva partecipazione è stato la cartina di tornasole di questo nuovo clima. Certamente molto resta da fare al riguardo, ma l'aver inserito nel nuovo Statuto un esponente delle aree non mediche nel Consiglio Direttivo credo rappresenti non solo un segnale forte e unico nel panorama scientifico italiano, ma anche la chiave di volta per un reale sviluppo e potenziamento delle loro professionalità.

Tra i progetti purtroppo solo avviati oppure neanche partiti, vorrei passare all'attenzione del nuovo Consiglio Direttivo quello relativo alla creazione di gruppi di lavoro interprofessionali su problematiche specifiche ed emergenti, come la standardizzazione dei modelli di esercizio fisico, specie nei pazienti più complessi, la ridefinizione dei programmi educazionali e di counseling, la proposta di un minimal data set per la cartella riabilitativa, la messa a punto e la raccolta sistematica di indicatori di processo e di efficacia, l'avvio di un programma di accreditamento professionale per gli operatori e, infine la proposta di costruire documenti di consenso su problemi da sempre irrisolti come l'idoneità lavorativa o alla guida dei cardiopatici. Infine, so già che il Presidente ha già messo in cantiere insieme 
con l'AMNCO un gruppo di lavoro su quello che reputo ancora il "problema dei problemi" già in qualche modo enfatizzato dalle LLGG ISS-PNLG-ASSR-GICR: la definizione di precisi percorsi riabilitativi e preventivi, sia organizzativi che assistenziali, appropriati nei cardiopatici post ischemici acuti. Anche in questo caso, uno sguardo all'Europa consente, sia pure nell'ambito di differenti sistemi sanitari, di partire da significative esperienze e modelli già in attuazione pratica: valga per tutti quello che è stato messo in atto a tale proposito in Germania, Gran Bretagna e soprattutto in Francia.

\section{La politica estera}

Con la FIC si è proseguito con un approccio molto collaborativo e leale: voglio sottolineare il grande impegno del Presidente Maseri sulla questione con la Fisiatria, anche se tuttora, nonostante il pronunciamento del Ministero della Salute indotto anche dal supporto degli amici di ConaCuore, persistono differenti atteggiamenti di alcune Regioni. Aldilà della vecchia e un po' stucchevole contrapposizione con la SIMFER, credo, e so che Carmine Riccio è d'accordo, che siano maturi i tempi per un confronto diretto tra cardiologi e fisiatri per il bene comune della riabilitazione in generale e di quella cardiologica in particolare, in un contesto economico e sociale difficile come quello attuale, in cui il rischio concreto è il "taglio" indiscriminato da parte del SSN delle prestazioni riabilitative.

Con Beppe Di Pasquale, la cui attiva partecipazione al nostro Congresso sottolinea la grande attenzione della FIC per il GICR e le sue scelte "politiche", la collaborazione è stata molto intensa e fruttuosa, sfociata nel nostro forte coinvolgimento e rappresentatività nella stesura del nuovo documento sulla Struttura e organizzazione funzionale della Cardiologia.

Per quanto riguarda i rapporti con ANMCO e SIC, la nostra posizione è ormai ben definita: tramontata l'idea di una casa comune, si è percorsa la strada della consueta collaborazione, testimoniata dalle tante iniziative comuni, e di una concreta integrazione tra GICR-IACPR e Area Prevenzione a partire dalla base e non solo dai vertici, in altre parole, integrando le iniziative locali, regionali e mettendo in stretta comunicazione i rispettivi soci e referenti. Con Stefano Urbinati, e anche su questo non c'erano dubbi, l'identità di vedute è totale.

Abbiamo poi, in modo coerente. imboccato con decisione la strada di una mutazione della nostra società con il nuovo statuto, che guarda ancor più all'Europa e che rappresenta uno strumento idoneo per intraprendere un percorso che porti il GICRIACPR ad essere nei fatti il più autorevole interlocutore italiano sulle problematiche della riabilitazione, della prevenzione e dell'epidemiologia, con una ancora più accentuata trasversalità con le altre società scientifiche.

Anche con l'EACPR la collaborazione è stata intensa e non va sottaciuto come la nostra forza associativa abbia contribuito, in modo consistente e aggiuntivo alla grande e indiscutibile personalità scientifica dei candidati italiani, all'elezione di Pantaleo Giannuzzi a President Elect, e all'affermazione nelle varie Section di Piepoli, Mezzani e Vanuzzo. Confido che il nuovo direttivo prosegua nella strada della nostra progressiva internazionalizzazione con una ancor più attiva partecipazione alla vita e agli eventi ed iniziative dell'EACPR. Non a caso EUROPREVENT è stato individuato come il palcoscenico più idoneo per presentare i risultati delle nostre survey.

\section{Conclusioni e ringraziamenti}

Non spetta certo a me dare un giudizio su questi due anni di lavoro, ma vi posso assicurare che il mio impegno è stato grande, molto maggiore di quanto a suo tempo avessi preventivato. Non me ne lamento, poiché esso è un indicatore che la nostra società sta crescendo in modo impetuoso e quindi pretende da chi la governa un forte impegno. Non credo che questa volontà difetti al caro amico Carmine Riccio e al suo capace Consiglio Direttivo: in questi due anni ho avuto il modo di apprezzarli tutti per il grande spirito collaborativo e ideativo e so di lasciare il Gruppo in mani ancora migliori delle nostre. Come Past Presidente ho la grande opportunità di continuare a collaborare "in presa diretta" alla vita del GICR-IACPR, portando con discrezione il mio piccolo contributo quando verrà richiesto e avendo ancora il piacere di stare "insieme" non solo con dei colleghi ma con sinceri amici, con i quali discutere di lavoro (ma anche di altro...) è sempre un grande arricchimento.

Voglio infine ringraziare tutti quelli che hanno contribuito a questi due anni di grande impegno: i soci innanzitutto, per il loro entusiasmo e la loro costante presenza alla vita associativa, i referenti delle Aree non mediche, che, pur tra grandi difficoltà organizzative (ed economiche...), hanno portato avanti con grande passione il loro mandato, gli amici delegati regionali, vera longa manus della società nelle Regioni, i comitati scientifici di ISYDE 2008 e di ICAROS che hanno contribuito in modo decisivo alla costruzione di quelle che rappresentano le principali iniziative scientifiche del GICR dai tempi del GOSPEL, l'impagabile segretaria-tesoriere Pasqualina Calisi, senza la cui professionalità e competenza specifica avrei fatto "saltare" la cassa del GICR e avrei ignorato l'esistenza di libri contabili, libro dei soci e quant' altro e che continuerò con piacere a "prestare" al nuovo Direttivo, gli Editors del Monaldi Archives for Chest Disease Carlo Vigorito e Carmine Chieffo che hanno continuato non solo a far uscire trimestralmente questa rivista con sempre maggior qualità e tempestività, ma ne hanno professionalizzato la struttura grazie anche ad una precisa contrattualizzazione con la casa editrice e, non ultimo, a non hanno mancato, nei momenti giusti, di fornirci i loro saggi consigli, 1"'esperto" amico Roberto Tramarin, la cui grande dedizione e competenza hanno reso possibile l'ideazione e il compimento di molte delle iniziative più significative del biennio.

Per ultimo, ma non certo per importanza, un grosso grazie di cuore alle nostre segretarie, temine veramente riduttivo per la qualità del lavoro svolto, Carla Chiavetta e Paola Biglino, la cui pazienza e sollecitudine nei miei confronti è stata veramente senza limiti. 


\title{
Saluto del Presidente 2008-2010
}

\section{Greetings from President 2008-2010}

\author{
Carmine Riccio
}

Monaldi Arch Chest Dis 2008; 70: 173-174.

Cari amici,

sono veramente felice ed orgoglioso di poter dare il mio contributo al processo di crescita della Cardiologia Riabilitativa in Italia, rivestendo per il prossimo biennio la carica di Presidente. Sono sicuro di poter contare su una squadra forte, costituita da persone come Roberto Carlon, Antonio Castello, Tommaso Diaco, Carlo Schweiger, e Pierluigi Temporelli un formidale mix di esperienza e passione.

Siamo appena rientrati da Ostuni, dove si è svolto il $9^{\circ}$ Congresso Nazionale del GICR-IACPR. L'elevato livello scientifico dei Simposi oltre alla crescente partecipazione alla vita associativa, fanno sicuramente ben sperare per il futuro, proseguendo nel costante processo di crescita che ha portato il GICRIACPR ad essere una delle più importanti società di settore federate nella FIC. Sicuramente ci muoviamo in un contesto difficile dove le risorse, sempre più ridotte, vengono indirizzate prevalentemente nella gestione della fase acuta. Per contrastare questa tendenza la Cardiologia Riabilitativa dovrà proseguire sulla strada dell'accreditamento all'eccellenza, puntando all'appropriatezza dei percorsi e alla verifica della qualità delle strutture e degli operatori.

Nello scorso biennio sono state definite una serie di iniziative allo scopo di conferire alla nostra società basi organizzative più solide, sono stati realizzati progetti di formazione e di ricerca che hanno garantito al GICR-IACPR crescita e visibilità. Ci si è resi conto poi che, per mantenere la nostra Società in linea con $\mathrm{i}$ tempi, era necessario procedere ad una modifica dello statuto. A partire dal nome, che ha affiancato allo storico GICR la sigla IACPR, Italian Association of Cardiovascular Prevention, Rehabilitation and Epidemiology, che rispecchia meglio il concetto di una società che vuole essere aperta a tutti coloro che si interessano di Prevenzione, dalla Epidemiologia alla Riabilitazione, e che intende far valere il suo ruolo anche in Europa, come partner dell'EACPR.

Quali strade percorrere nel prossimo biennio?

Il GICR-IACPR dovrà mantenere e rinforzare il suo ruolo nel panorama delle società scientifiche italiane. Sarà garantito un pieno e leale appoggio alla Federazione Italiana di Cardiologia, così come sancito a chiare lettere già nello statuto. È in avanzata fase di realizzazione la revisione del documento su "Struttura ed Organizzazione funzionale della Cardiologia", alla cui stesura il GICR-IACPR ha contribuito in misura sostanziale.

Saremo attenti interlocutori dell'Istituto Superiore di Sanità per eventuali iniziative in ambito preventivo.

Per quanto riguarda l'ANMCO, sapete tutti quanto io vi sia legato; gli anni trascorsi in Area Prevenzione sono stati una straordinaria esperienza che sicuramente mi sarà di grande aiuto in questo biennio, oltre ad arricchirmi dell'amicizia di persone come Massimo Uguccioni, Furio Colivicchi, Alberto Genovesi, Ilio Faggiano e tanti altri che, al di là delle sigle, saranno per me sempre punti di riferimento. In un certo momento "storico" ci era sembrato possibile ipotizzare una "casa comune" che raccogliesse le varie anime della prevenzione. Abbiamo capito che oggi questo non è possibile, o almeno non lo è per come ci eravamo prefigurati. Ora abbiamo tutti le idee più chiare su quali scenari di collaborazione saranno possibili. Sono sicuro che in un momento in cui abbiamo due grandi amici, visceralmente legati alla storia della nostra società, come Salvatore Pirelli alla presidenza dell' ANMCO e Stefano Urbinati come Chairman dell'Area Prevenzione, sapremo sviluppare insieme progetti formativi e di ricerca di grande qualità e lavorare insieme per la preparazione della IV Conferenza Nazionale di Prevenzione Cardiovascolare prevista per il 2010. Come fase di ulteriore crescita del GICR-IACPR è giunto poi il momento di definire con l'ANMCO percorsi appropriati in prevenzione secondaria, abbandonando posizioni oltranziste ed insostenibili, identificando quei pazienti per i quali il passaggio in un centro di Cardiologia Riabilitativa è assolutamente raccomandabile, rispetto ad altri, a più a basso rischio e a minor grado di complessità, per i quali è sufficiente un pacchetto concordato di misure di prevenzione secondaria.

Ovviamente il GICR-IACPR avrà uguale attenzione per tutte le altre società scientifiche, rendendosi promotore o attivo partecipe di progetti formativi, organizzativi o di ricerca in ambito formativo.

Dovremo rinforzare il nostro ruolo di ponte tra l'acuzie ed il territorio. Con la Medicina Generale andranno analizzati compiutamente le criticità che impediscono l'attuazione delle misure di prevenzione secondaria, basate sull'evidenza ma fortemente disattese nella pratica clinica. Piuttosto che continuare a disperarsi per l'oscurità, leggi dati Euroaspire, un proverbio cinese suggerisce di provare ad accendere una candela. Sono già in avanzata fase di studio percorsi condivisi per la gestione integrata del paziente reduce da un evento acuto coronarico, a partire dalla criticità del momento della dimissione. Il simposio congiunto organizzato ad Ostuni può costituire un interessante modello di lavoro.

Dovremo poi impegnarci per garantire ai pazienti una corretta informazione orientata alla prevenzione. Se oggi un paziente si mette sul Web alla ricerca di notizie relative, per esempio, ad un corretta alimentazione, trova molto poco sui siti delle società scientifiche italiane, indirizzati per la maggior parte all'informa- 
zione della comunità cardiologica. L'esatto opposto di quello che succede negli Stati Uniti, dove il sito delle società scientifiche sono una miniera di informazioni per il cittadino-paziente. Oltre al Sito, penso ad opuscoli o periodici che diano risposte a quesiti che quotidianamente i cittadini-pazienti ci formulano. Sinergie con la Fondazione per il tuo cuore-HCF saranno ricercate per raggiungere questo obiettivo, ma sarà fondamentale anche l'apporto delle Associazioni di pazienti, come Conacuore, che svolgono un ruolo strategico in ambito preventivo nel tessuto sociale.

Sempre più vorrei che passasse il messaggio che la mission della cardiologia riabilitativa sia non solo di curare ma di "prendersi cura" dei pazienti.

Con le Aziende Farmaceutiche il GICR-IACPR ha sempre mantenuto rapporti sereni improntati alla reciproca collaborazione. I progetti formativi realizzati, il sostegno alla rivista ed al sito sono stati portati avanti sulla base di una progettualità scientifica, nel rispetto di ineccepibili norme di etica e trasparenza. Continueremo su questa strada, garantendo l'entusiasmo e la preparazione di una società scientifica giovane, con un apparato burocratico snello ed adeguato a rispondere in tempi rapidi alle necessità del mondo dell'industria.

Per quanto riguarda la politica interna, il GICRIACPR continuerà a promuovere iniziative nei settori dove è tradizionalmente più forte. Penso alla formazione in cui ormai il marchio GICR è considerato un brand di qualità; già sono allo studio una serie di proposte che, come è ormai tradizione, sperimenteranno anche percorsi formativi innovativi.

Penso anche alla ricerca: nello scorso biennio abbiamo percepito quale ruolo possono avere le survey, uno strumento di elevatissimo rapporto costoefficacia, vedi ISYDE e ICAROS e di cui sicuramente il gruppo si avvarrà anche per il futuro. Sarebbe poi il momento di proporre studi di fisiopatologia, utilizzando per esempio il know-how della Cardiologia Riabilitativa sugli effetti del training fisico, sull'endotelio o sul test cardiopolmonare.

Arriviamo al sito GICR-IACPR, che oggi ritengo tra i migliori siti delle società scientifiche italiane, e di questo bisogna dire grazie a Roberto Tramarin e a quanti hanno collaborato con lui; il lavoro fatto in questo biennio è stato impressionante per qualità e quantità di progetti avviati e portati a termine, pensiamo all'Isyde, all'Icaros, proprio in questi giorni in rampa di lancio. Il sito però funziona fin quando c'è interesse e partecipazione. Vorrei quindi che fosse sentito da tutti i soci come parte integrante della vita associativa, inviando contributi, aggiornando dati, segnalando articoli interessanti, proponendo magari delle review ragionate. E questo invito vale ovviamente anche per la nostra rivista, il Monaldi Archives, biglietto di presentazione scientifica e culturale del GICR-IACPR, una rivista indicizzata che pochi altri possono vantare. Bisogna ringraziare Carmine Chieffo e Carlo Vigorito, i due direttori, la cui dedizione, competenza e tenacia ha consentito alla rivista di essere sulle nostre scrivanie ogni tre mesi, superando ostacoli organizzativi ed economici non semplici. Alla luce delle nuove tecnologie, bisognerà ricercare sistemi che ci consentano di garantire alla rivista una diffusione più mirata con un risparmio dei costi.

Va perseguita la multidisciplinarietà del gruppo. Moltissimo è stato fatto nello scorso biennio per dare spazio e visibilità alle aree non mediche. Si prose- guirà alla luce anche delle modifiche dello statuto su questa strada. Invito quindi i referenti, i cui nomi sono stati proposti dalle Aree e immediatamente fatti propri dal Consiglio Direttivo, a proporsi costantemente, a portare le istanze delle professionalità che rappresentano. Troveranno nel Consiglio Direttivo attenzione alle loro proposte.

Sta partendo in questi giorni la lettera di incarico per i Delegati Regionali, il cui ruolo è ritenuto dal CD strategico per le finalità del gruppo. In un momento di regionalizzazione dei modelli sanitari è indispensabile che i singoli Delegati Regionali, così come da mandato, ricerchino rapporti con le Istituzioni. Laddove si dovessero creare delle specifiche problematiche, il GICR-IACPR sarà disponibile ad affiancare il Delegato Regionale. È stato identificato un referente per i DR all'interno del CD, nella figura di Tommaso Diaco, la cui esperienza maturata negli ultimi 4 anni, sarà messa a disposizione di tutti i DR per affrontare al meglio il loro compito.

Il GICR-IACPR per realizzare questi progetti ha bisogno però di una società forte e la forza di una società nasce dalla partecipazione dei soci sia in termini di contributi di idee e di progetti, sia in termini di iscrizioni. Mi sembra assurdo che in alcuni momenti, lontani da aspirazioni elettorali, siano in regola con le iscrizioni al gruppo un numero inferiore di persone rispetto ai centri censiti. Faremo di tutto per facilitare il pagamento delle quote, ipotizzeremo sistemi di incentivazione delle quote, dopodiché però trarremo le fila e garantiremo accesso e disponibilità ai "benefici" dei gruppo solo a chi avrà dimostrato di voler "far parte del gruppo".

\section{Ringraziamenti}

Prima di tutto, anche per ricollegarmi idealmente con l'inizio di questa mia avventura, un grazie a Carmine Chieffo, che per me sarà sempre il grande capo, senza il quale, sicuramente, non sarei qui a portarvi il saluto come vostro futuro presidente.

Un grazie va a Francesco Fattirolli e a Lello Griffo; due amici veri, da loro ho cercato di rubare qualche segreto del mestiere, a loro chiederò collaborazione anche in futuro.

Ringrazio tutti quelli con cui lavoro in ospedale a Caserta, da Ornella Zanni a tutti i fisioterapisti, ai colleghi, in particolare a Angelo Cardillo, Maurizio Di Stasio, Antonio Palermo e Donato Soviero, la cui pazienza e disponibilità mi ha consentito di ritagliarmi del tempo per il GICR-IACPR.

Grazie a Paolo Golino che mi ha sempre incitato ad andare avanti su questa strada, garantendomi spazi di autonomia e preziosi consigli. Un abbraccio affettuoso al professore Corrado Tritto, che in questi anni ha costituito per me un chiaro punto di riferimento, un concentrato di saggezza ed equilibrio.

Un grazie a tutti quelli che mi sono stati vicini in un certo momento della mia vita.

Infine grazie a Stefania, Gabriele e Benedetta a cui devo delle scuse per il tempo che gli sottraggo: forse avrebbero desiderato un marito ed un papà un po' meno presidente, ma un po' più presente.

Un grazie a tutti voi per il sostegno e la simpatia con cui mi avete accolto. Mi sento di garantirvi grande entusiasmo e disponibilità e un pizzico di napoletanità per raggiungere insieme gli obiettivi prefissati. 